\title{
Side Information Refinement for Long Duration GOPs in DVC
}

\author{
Giovanni Petrazzuoli, Thomas Maugey, Marco Cagnazzo, Béatrice Pesquet-Popescu \\ Signal and Image Processing Department, Institut TELECOM - TELECOM ParisTech \\ 46 rue Barrault, F-75634 Paris Cedex 13, FRANCE \\ \{petrazzu, maugey, cagnazzo, pesquet\} @telecom-paristech.fr
}

\begin{abstract}
Side information generation is a critical step in distributed video coding systems. This is performed by using motion compensated temporal interpolation between two or more key frames (KFs). However, when the temporal distance between key frames increases (i.e. when the GOP size becomes large), the linear interpolation becomes less effective. In a previous work we showed that this problem can be mitigated by using high order interpolation. Now, in the case of long duration GOP, state-ofthe-art algorithms propose a hierarchical algorithm for side information generation. By using this procedure, the quality of the central interpolated image in a GOP is consistently worse than images closer to the KFs. In this paper we propose a refinement of the central WZFs by higher order interpolation of the already decoded WZFs, that are closer to the WZF to be estimated. So we reduce the fluctuation of side information quality, with a beneficial impact on final rate-distortion characteristics of the system. The experimental results show an improvement on the SI up to $2.71 \mathrm{~dB}$ with respect the state-of-the-art and a global improvement of the PSNR on the decoded frames up to $0.71 \mathrm{~dB}$ and a bit rate reduction up to $15 \%$.
\end{abstract}

\section{INTRODUCTION}

In recent years, several applications, such as sensor networks, require a low-complexity encoder due to limited resources in terms of available memory and power at the encoder. Standard video techniques, created for the broadcast transmission, provide a complex encoder, but a very simple decoder. The complexity of the encoder for standard video techniques is due to motion estimation and compensation (i.e. the joint encoding of temporally adjacent frames). The purpose of distributed video coding (DVC) [1] is to move complexity from the encoder to the decoder. To simplify the encoder we can not exploit the dependency between temporally adjacent frames at the encoder, but only at the decoder. Wyner and Ziv [2] have shown that there is no loss of rate-distortion performance between jointly coding and distributed coding if the sources are jointly gaussian. In distributed video coding the video sequence is split into key frames (KFs) and WynerZiv frames (WZFs). The distance between two KFs is called GOP size. So, a GOP of size equal to $N$ consists in having one $\mathrm{KF}$ and $N-1 \mathrm{WZFs}$. We consider the following frameworks proposed in [3]: the KFs are intra-coded (i.e. we exploit only the spatial dependence in the frame and not the

MMSP'10, October 4-6, 2010, Saint-Malo, France. 978-1-4244-8112-5/10/\$26.00 (c)2010 IEEE. temporal correlation between adjacent frames). The WZFs are transformed by a DCT and after turbo-encoded: the output of the turbo encoder consists in a systematic part (the true WZF) and in its parity bits. We send at the decoder only the parity bits. At the decoder, an estimation of the WZF is produced by interpolating the adjacent KFs. This estimation may be considered as a noisy version of the true WZF. The turbodecoder corrects this error by using the parity bits send by the turbo encoder. It can happen, however, that parity bits sent are not sufficient to ensure a small bit error rate. Indeed, the structure of this video coder is provided with a return channel to request to the turbo encoder more parity bits. So, also if the KFs and WZFs are dependent sources (because they are adjacent frames), we encode them separately. The dependence is exploited only at the decoder.

It is very important to correctly estimate the Wyner-Ziv frame because it allows us to send less parity bits to correct it. The GOP size is usually equal to 2 in the state of the art solutions. In this case the estimation procedure is very simple: the odd frames are KFs, the even frames are WZFs and for each WZFs we interpolate the two (or more) closest KFs.

It can also be interesting to study cases where the GOP size is more than 2: the number of WZFs is larger than the KFs, and then the encoding requires a bit rate smaller than when the GOP size is equal to 2. In [4], [5], [6] it is proposed to use a hierarchical structure for estimating and decoding the WZFs for GOP size equal to 4, where at first the central WZF of the GOP is estimated and decoded. Then, since it is available at the decoder, it can be used for interpolating the other WZFs, called lateral WZFs. This causes, however, large fluctuations in PSNR of the SI for each WZF, because the lateral WZFs are better estimated than the central one. As consequence, the rate required for decoding the central WZF is greater than of the lateral ones. So, we propose to refine the estimation of the central WZF by interpolating the lateral decoded WZFs, but without demanding new parity bits to the turbo encoder and only using those sent at the first step. The coder used in this paper is shown in the Fig. 1, where the image interpolation block uses as input the previously decoded WZFs (the light blue dashed arrow). Our algorithm improves both PSNR on SI and the RD performance with respect to a simple hierarchical coding. Our algorithm is also extended to the case where the GOP size is equal to 8 .

This paper is organized as follows: in section 2 we describe 


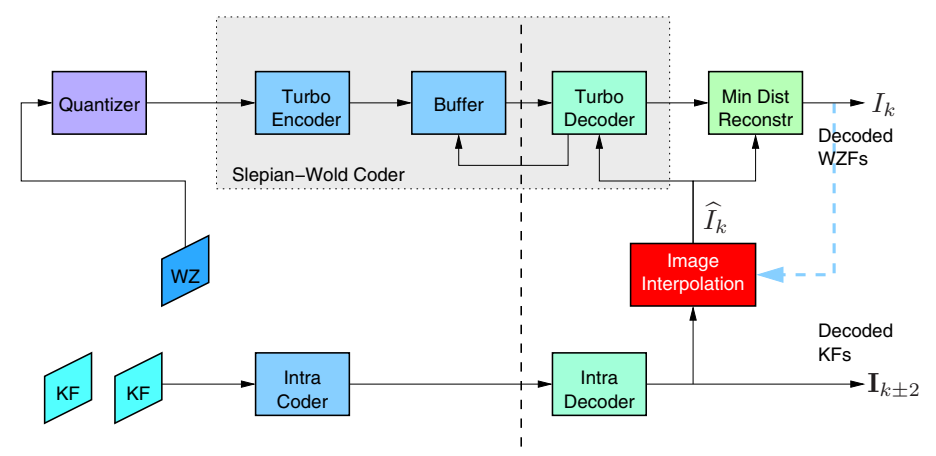

Fig. 1. Codec used in this paper

the methods for generating the SI and the state-of-the-art for hierarchical estimation and decoding. In section 3 we describe our method for the refinement of the SI. In section 4 we show the experimental results. In section 5 we discuss conclusions and future works.

\section{REFERENCE METHOD}

The algorithm for the motion interpolation given by DISCOVER [7] is the most popular in the context of DVC. Let $X_{k}$ be the WZF that we want to estimate. This method consists by linear interpolating the two closest frames available to $X_{k}$ at the decoder. Let $X_{b}$ be the closest previous available frame and $X_{f}$ the closest following available frame ${ }^{1}$. It consists of the following steps:

- The motion vector field (MVF) from $X_{f}$ to $X_{b}$ is estimated.

- This MVF is split into a forward MVF and into a backward MVF.

- A median filter is performed over the two MVFs.

- The estimation of the WZF is the average of $X_{f}$ compensated by the forward MVF and $X_{b}$ compensated by the backward MVF

We have proposed an improvement of this algorithm in [8]. A higher order motion interpolation (HOMI) is performed by using the closest four frames to $X_{k}$ for estimating it. Let $X_{a}$ and $X_{b}$ be the two closest previous available frames and $X_{f}$ and $X_{g}$ be the two closest following available frames. It consists in the following steps:

- The backward and the forward MVFs $\mathbf{u}$ and $\mathbf{w}$ are estimated by using DISCOVER

- The refinement $\delta \mathbf{u}$ [or $\delta \mathbf{w}$ ], that minimizes the following function is found.

$$
J(\delta \mathbf{u})=\sum_{\mathbf{q}}\left|B_{f}^{\mathbf{p}+\mathbf{u}(\mathbf{p})}(\mathbf{q})-B_{g}^{\mathbf{p}+t \mathbf{u}(\mathbf{p})+\delta \mathbf{u}}(\mathbf{q})\right|+\lambda\|\delta \mathbf{u}\|
$$

where $B_{f}^{\mathrm{p}}$ is the macroblock (MB) of the frame $X_{f}$ centered in $\mathbf{p}$ and $t$ is defined as $1+d$ where $d$ is the

\footnotetext{
${ }^{1}$ Let $X_{i}$ be the frames at instant $i$
}

distance (in number of frames) between $X_{f}$ and $X_{g}$ (i.e. $g-f)$.

- By interpolating the four positions $\mathbf{p}+t \mathbf{w}(\mathbf{p})+\delta \mathbf{w}, \mathbf{p}+$ $\mathbf{w}(\mathbf{p}), \mathbf{p}+\mathbf{u}(\mathbf{p}), \mathbf{p}+t \mathbf{u}(\mathbf{p})+\delta \mathbf{u}$ in the instants $a, b, f$ and $g$, we found the position $\widehat{\mathbf{p}}$ in $k$.

- The new motion vectors, centered in $\mathbf{p}$, are defined as $\widehat{\mathbf{w}}=\mathbf{p}+\mathbf{w}(\mathbf{p})-\widehat{\mathbf{p}}$ and $\widehat{\mathbf{u}}=\mathbf{p}+\mathbf{u}(\mathbf{p})-\widehat{\mathbf{p}}$

- The estimation of the WZF is the average of $X_{f}$ compensated by $\widehat{\mathbf{w}}$ and $X_{b}$ compensated by $\widehat{\mathbf{u}}$

Note that $X_{a}, X_{b}, X_{f}$ and $X_{g}$ are not necessarily KFs, but they can be also already decoded WZFs and now available at the decoder.

For GOP size equal to 2, the decoding procedure is very simple $^{2}$. If $I_{k}$ is the WZF that we want to estimate, it is sufficient to interpolate the KFs $\mathbf{I}_{k+1}$ and $\mathbf{I}_{k-1}$ (or also $\mathbf{I}_{k-3}$ and $\mathbf{I}_{k+3}$ if we want to apply the HOMI method). The decoding procedure becomes more difficult if GOP size is greater than 2 . For simplicity only situations where the GOP size is a power of two (i.e. 2, 4, 8) [4] are analyzed. For GOP size different to a power of two, see [5]. Let GOP size be equal to 4 . The hierarchical structure of estimation and decoding is shown in [4]. It consists in the following steps

1) The central WZF at instant $k$ is estimated by using the KFs $\mathbf{I}_{k-2}$ and $\mathbf{I}_{k+2}$. This estimation is called $\widehat{I}_{k}$ (Fig. 2(a)) and it is corrected by using $R_{k}$ parity bits. The decoded WZF is called $I_{k}$

2) The lateral WZF at instant $k-1$ is estimated by using the KF $\mathbf{I}_{k-2}$ and the decoded WZF $I_{k}$ (Fig. 2(b)) and the lateral one at instant $k+1$ is estimated by using the KF $\mathbf{I}_{k+2}$ and the decoded WZF $I_{k}$ (Fig. 2(c)). The estimation are corrected by a turbo-decoder by using, respectively, $R_{k-1}$ and $R_{k+1}$ parity bits. The decoded WZFs are called $I_{k-1}$ and $I_{k+1}$

In this manner, the PSNR of the WZF estimation with respect to the real one is better for the lateral WZF because they are better estimated. In fact, for the central WZF we interpolate frames that have a distance of two from the reference, while for the lateral WZF we interpolate frames that have a distance of one from the reference. Therefore, we observe that the PSNR is floating also on the decoded frames (see Fig. 3). In fact, for book arrival sequence we observe that the average PSNR of the central decoded WZFs is 36.70 $\mathrm{dB}$ with a bit rate of $23.24 \mathrm{kbps}$ (we consider only the parity bits), while the average PSNR of the lateral decoded WZFs is $38.13 \mathrm{~dB}$ with a bit rate of $16.70 \mathrm{kbps}$.

Then, our target in this work is to improve the central WZF in the context of hierarchical coding.

\section{PROPOSED METHOD}

Given the large difference of PSNR between the central decoded WZF and lateral decoded WZF when the GOP size is equal to 4 , we propose to refine the estimation of the central

\footnotetext{
${ }^{2}$ In the following the decoded WZFs are shown in italics (e.g. $I_{k}$ ). The KFs are shown in bold (e.g. $\mathbf{I}_{k-1}$ ). The side information (i.e. the WZF estimation) is indicated with an hat (e.g. $\left.\widehat{I}_{k}\right)$
} 


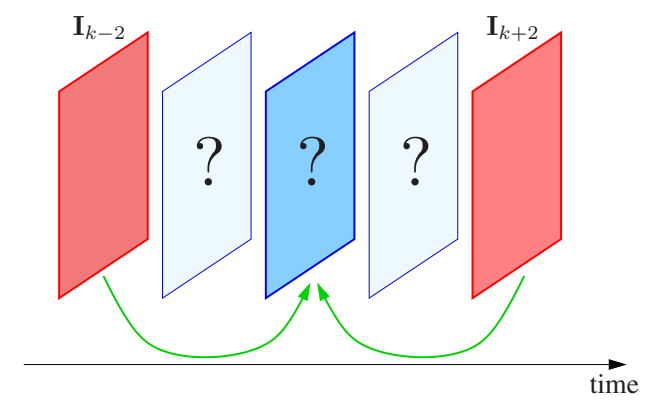

(a)

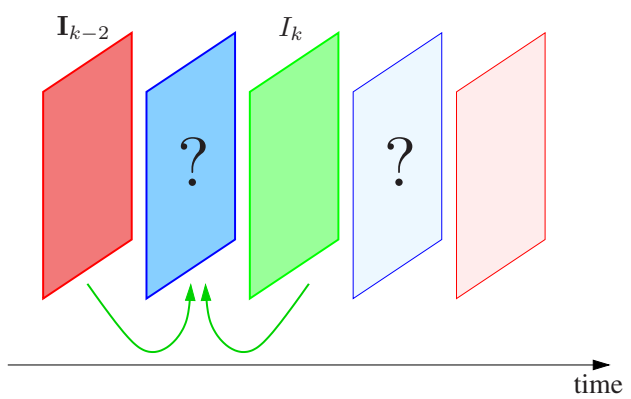

(b)

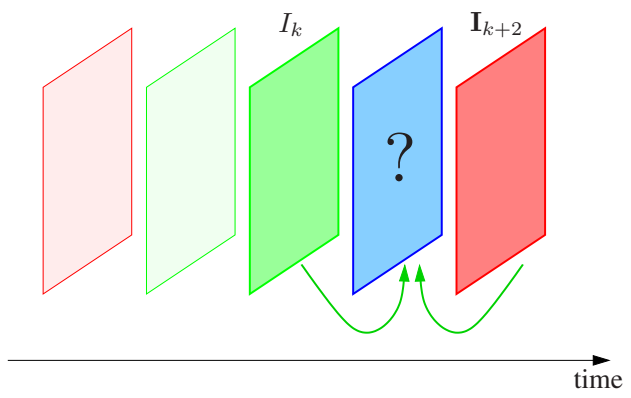

(c)

Fig. 2. WZ frame estimation for GOP size $=4$ (the KFs are in red, the WZFs are in blue, the already decoded WZFs are in green): at first we estimate $I_{k}$ (2(a)), after by it, we estimate $I_{k-1}(2(\mathrm{~b}))$ and $I_{k+1}(2(\mathrm{c}))$

WZF by interpolating the lateral WZFs previously decoded and now available at the decoder. The proposed algorithm uses interpolation of higher order (HOMI) [8] and consists in the following steps:

1) The estimation of the central WZF, $\widehat{I}_{k}$, is obtained by interpolating with HOMI the KFs $\mathbf{I}_{k-6}, \mathbf{I}_{k-2}, \mathbf{I}_{k+2}$ and $\mathbf{I}_{k+6}$ (see Fig. 4(a)). It is turbo encoded by using $R_{k}$ parity bits. Let $I_{k}$ be the decoded WZF.

2) The estimation of the lateral WZF, $\widehat{I}_{k-1}$, is obtained by interpolating with HOMI the decoded central WZF of the previous GOP $I_{k-4}$, the $\mathrm{KF} \mathbf{I}_{k-2}$, the decoded WZF $I_{k}$ and the KF $\mathbf{I}_{k+2}$ (see Fig. 4(b)). Likewise, the estimation of the lateral WZF $\widehat{I}_{k+1}$ is obtained by interpolating by HOMI the $\mathrm{KF} \mathbf{I}_{k-2}$, the decoded $I_{k}$, the KF $\mathbf{I}_{k+2}$, and the decoded central WZF of the next GOP $I_{k+4}$ (see Fig. 4(c)). The two lateral WZFs are turbo-decoded by using, respectively, $R_{k-1}$ and $R_{k+1}$ parity bits. Let $I_{k-1}$ and $I_{k+1}$ be the decoded WZFs.

3) Now, also the decoded WZF $I_{k-1}$ and $I_{k+1}$ are available at the decoder. Then, we can refine the estimation of $I_{k}$,

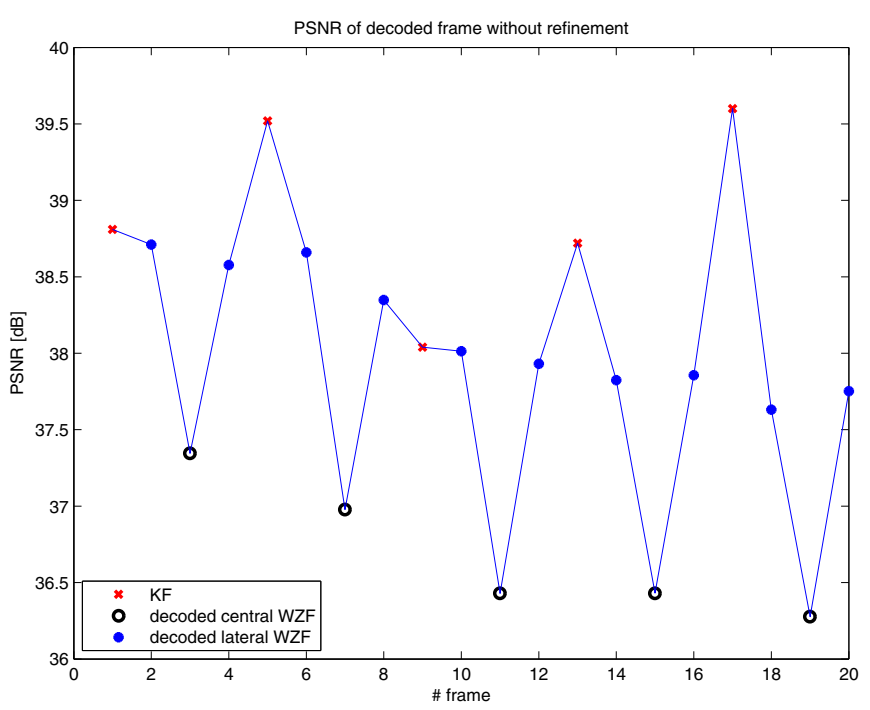

Fig. 3. PSNR of decoded frames for book arrival, $Q P=31$, GOP size $=4$

by using them. The WZF $I_{k}$ is closer to $I_{k-1}$ and $I_{k+1}$ than $I_{k-2}$ and $I_{k+2}$. So, we expect that the estimation by using the first is better than by using the latter. More precisely, the WZF $I_{k}$ is refined by using the KFs $\mathbf{I}_{k-2}$, the decoded WZFs $I_{k-1}, I_{k+1}$ and the KF $\mathbf{I}_{k+2}$ (see Fig. 4(d)). This new estimation is now turbo-decoded by using the same parity bits of the step 1 . Then, it is not required to resend the parity bits. The decoder only uses parity bits previously sent (see also Tab. I).

The turbo-decoding of the WZF $I_{k}$ at step 3 is sub-optimal. In our algorithm of turbo-decoding the WZF are transformed by a $4 \times 4$ DCT. The rate allocation for each band is dependent of the estimation of the parameter $\alpha$ of the laplacian pdf that models the estimation error $X-Y$, where $X$ is the true WZF and $Y$ is its estimation [9], [10]. From step 1 to step 3 the side information $Y$ is changed, and so is it for the parameter $\alpha$. Moreover, one can not ask again the turbo coder to reallocate parity bits for each band for the new side information, as this would significantly increase the total bit rate. Nevertheless, at the same total bit rate we expect that the average PSNR of the decoded WZFs increases after refinement.

However, the computational effort at the decoder is increased: in fact, in the proposed method, the side information must be generated twice and must be turbo decoded twice, but only for the central WZFs.

This method can be extended to the case of GOP size of 8 . The extension is straightforward but heavy to describe, so we summarize it in the Tab. II.

\section{EXPERIMENTAL RESULTS}

Several experiments have been carried out in order to tune and validate the proposed method. At first we compared the HOMI method (hierarchical coding before refinement) and the proposed method with respect to the DISCOVER method (hierarchical coding without refinement) in terms of quality 


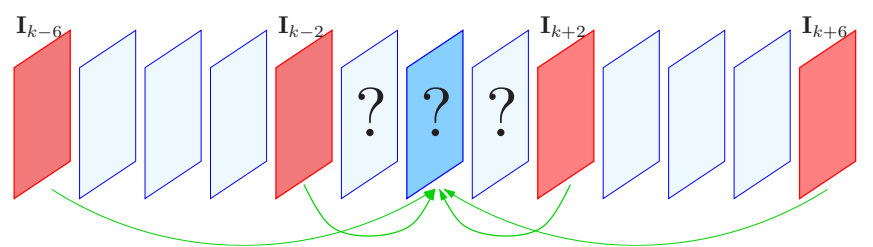

(a)

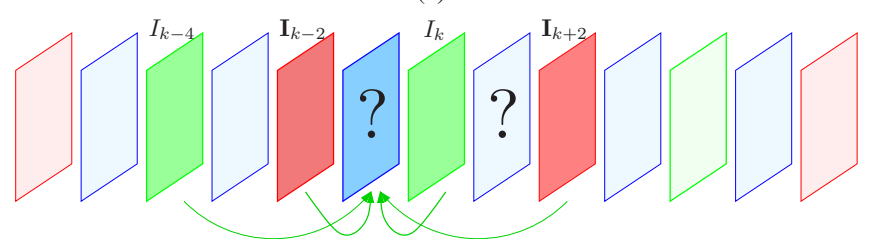

(b)

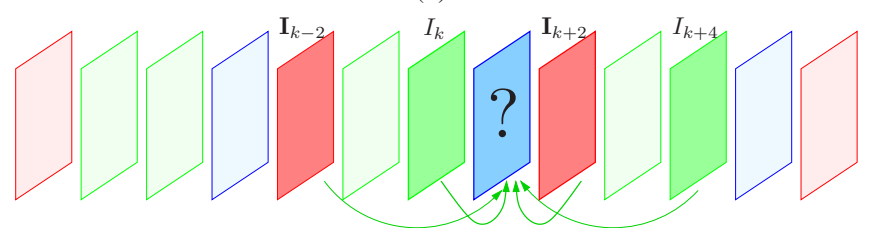

(c)

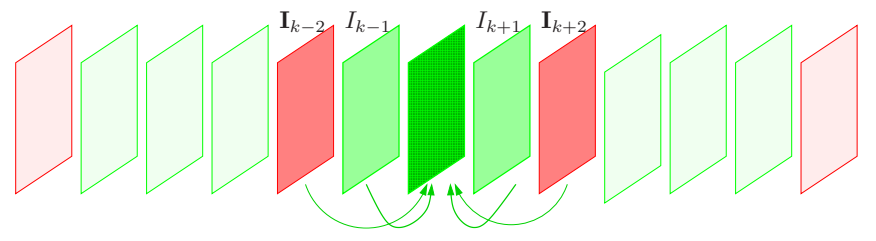

(d)

Fig. 4. WZ frame estimation for GOP size $=4$ (the KFs are in red, the WZFs are in green, the already decoded WZF are in green. the WZF to be refined is in dark green): at first we estimate $I_{k}$ (4(a)), after by it, we estimate $I_{k-1}(4(\mathrm{~b}))$ and $I_{k+1}(4(\mathrm{c}))$. Finally we refine the central $I_{k}(4(\mathrm{~d}))$

\begin{tabular}{|l|l|c|c|c|c|}
\hline step & SI construction & \multicolumn{5}{|c|}{ references } \\
\hline \hline \multicolumn{5}{|c|}{ GOP size $=4$} \\
\hline 1 & $\widehat{I}_{k}$ & $\mathbf{I}_{k-6}$ & $\mathbf{I}_{k-2}$ & $\mathbf{I}_{k+2}$ & $\mathbf{I}_{k+6}$ \\
\hline 2 & $\widehat{I}_{k-1}$ & $I_{k-4}$ & $\mathbf{I}_{k-2}$ & $I_{k}$ & $\mathbf{I}_{k+2}$ \\
& $\widehat{I}_{k+1}$ & $\mathbf{I}_{k-2}$ & $I_{k}$ & $\mathbf{I}_{k+2}$ & $I_{k+4}$ \\
\hline 3 & $\widehat{I}_{k}$ & $\mathbf{I}_{k-2}$ & $I_{k-1}$ & $I_{k+1}$ & $\mathbf{I}_{k+2}$ \\
\hline
\end{tabular}

TABLE I

FRAMES USED FOR THE WZF ESTIMATION FOR GOP SIZE EQUAL TO 4: KFS ARE IN BOLD AND THE DECODED WZFS ARE IN ITALIC.

of side information. After we perform a complete end-to-end coding analysis to evaluate the rate distortion performance.

The KFs are coded with H.264 in INTRA mode for different QP. The test sequences are book arrival, ballet, jungle and breakdancer.

\section{A. Side information construction}

Obviously the value of $\lambda$, defined in Eq. 1, changes for each step, depending of the distance of the frames that we interpolate. The optimal value of $\lambda$ in Eq. 1 is found by maximizing the average PSNR between the WZF estimation and the true one. The optimal value of $\lambda$ are already shown in [8], with the exception of cases where the frame to interpolate are not equally spaced (see Tab. III).

\begin{tabular}{|l|l|c|c|c|c|}
\hline step & SI construction & \multicolumn{4}{|c|}{ references } \\
\hline \hline \multicolumn{5}{|c|}{ GOP size $=8$} \\
\hline 1 & $\widehat{I}_{k}$ & $\mathbf{I}_{k-12}$ & $\mathbf{I}_{k-4}$ & $\mathbf{I}_{k+4}$ & $\mathbf{I}_{k+12}$ \\
\hline 2 & $\widehat{I}_{k-2}$ & $I_{k-8}$ & $\mathbf{I}_{k-4}$ & $I_{k}$ & $\mathbf{I}_{k+4}$ \\
& $\widehat{I}_{k+2}$ & $\mathbf{I}_{k-4}$ & $I_{k}$ & $\mathbf{I}_{k+4}$ & $I_{k+8}$ \\
\hline \multirow{3}{*}{3} & $\widehat{I}_{k-3}$ & $I_{k-6}$ & $\mathbf{I}_{k-4}$ & $I_{k-2}$ & $I_{k}$ \\
& $\widehat{I}_{k-1}$ & $\mathbf{I}_{k-4}$ & $I_{k-2}$ & $I_{k}$ & $I_{k+2}$ \\
& $\widehat{I}_{k+1}$ & $I_{k-2}$ & $I_{k}$ & $I_{k+2}$ & $\mathbf{I}_{k+4}$ \\
& $\widehat{I}_{k+3}$ & $I_{k}$ & $I_{k+2}$ & $\mathbf{I}_{k+4}$ & $I_{k+6}$ \\
\hline \multirow{3}{*}{4} & $\widehat{I}_{k-2}$ & $\widehat{I}_{k-4}$ & $I_{k-3}$ & $I_{k-1}$ & $I_{k}$ \\
& $\widehat{I}_{k+2}$ & $I_{k}$ & $I_{k+1}$ & $I_{k+3}$ & $\mathbf{I}_{k+4}$ \\
& $\widehat{I}_{k}$ & $I_{k-2}$ & $I_{k-1}$ & $I_{k+1}$ & $I_{k+2}$ \\
\hline
\end{tabular}

TABLE II

FRAMES USED FOR THE WZF ESTIMATION FOR GOP SIZE EQUAL TO 8: KFS ARE IN BOLD AND THE DECODED WZFS ARE IN ITALIC.

\begin{tabular}{|c|c|}
\hline \multicolumn{2}{|c|}{ GOP size $=4$} \\
\hline$\lambda_{1}$ & 20 \\
$\lambda_{2}$ & 50 \\
$\lambda_{3}$ & 40 \\
\hline \hline \multicolumn{2}{|c|}{ GOP size $=8$} \\
\hline$\lambda_{1}$ & 0 \\
$\lambda_{2}$ & 20 \\
$\lambda_{3}$ & 50 \\
$\lambda_{4}$ & 40 \\
\hline
\end{tabular}

TABLE III

VALUE OF $\lambda_{\text {OPT }}$ FOR THE DIFFERENT STEPS FOR GOP SIZE EQUAL TO 4 AND 8

The improvement in terms of PSNR of the side information with respect to DISCOVER are shown in Tab. IV for GOP size equal to 4 and in Tab. V for GOP size equal to 8 . We show the improvement achieved by HOMI method and by the proposed method in this paper with a hierarchical structure of decoding and estimation. We observe that we gain on the side information up to $1.99 \mathrm{~dB}$ for GOP size equal to 4 and up to 2.71 for GOP size equal to 8 . The gains for GOP size equal to 8 are most significant because for the refinement of the central WZF we use frames much closer than the first step (the distance of the frames to interpolate lowers from 8 to 2) and furthermore we refine 3 WZFs of 8 frames in the GOP. In Fig. 5, we show an estimated central WZF for book arrival sequence when the GOP size is equal to 8 . The estimated frame in step 1 is shown in Fig. 5(a) where we note that HOMI with a simple hierarchical estimation can not reconstruct the man on the right because the frames used for interpolation are too far apart. On the contrary with the proposed method (see Fig. 5(b)) the procedure of refinement correctly reconstructs the man on the right.

\section{B. RD performance}

The last experiment consisted in computing end-to-end performances (i.e. rate reduction and PSNR improvement) of the proposed techniques when inserted into a complete DVC coder like the one in Fig. 1, by using the the Bjontegaard metric [11]. This results are shown in Tab. VI for GOP size 


\begin{tabular}{|c|c|c|c|c|}
\hline QP & book arrival & ballet & jungle & breakdancer \\
\hline \multicolumn{5}{|c|}{ HOMI } \\
\hline \hline 31 & 0.40 & 0.33 & 0.23 & 0.08 \\
34 & 0.38 & 0.30 & 0.22 & 0.07 \\
37 & 0.33 & 0.22 & 0.21 & 0.08 \\
40 & 0.28 & 0.19 & 0.19 & 0.06 \\
\hline \hline \multicolumn{5}{|c|}{ proposed } \\
\hline 31 & 1.99 & 1.59 & 1.66 & 0.76 \\
34 & 1.80 & 1.64 & 1.45 & 0.76 \\
37 & 1.77 & 1.57 & 1.36 & 0.51 \\
40 & 1.71 & 1.42 & 1.27 & 0.81 \\
\hline
\end{tabular}

TABLE IV

$\Delta_{\text {PSNR }}[\mathrm{DB}]$ ON THE SIDE INFORMATION FOR GOP SIZE EQUAL TO 4 WITH RESPECT TO DISCOVER FOR HOMI AND FOR PROPOSED METHOD

\begin{tabular}{|c|c|c|c|c|}
\hline QP & book arrival & ballet & jungle & breakdancer \\
\hline \multicolumn{5}{|c|}{ HOMI } \\
\hline \hline 31 & 0.45 & 0.32 & 0.31 & 0.10 \\
34 & 0.46 & 0.33 & 0.29 & 0.09 \\
37 & 0.40 & 0.36 & 0.29 & 0.10 \\
40 & 0.32 & 0.27 & 0.26 & 0.10 \\
\hline \hline \multicolumn{5}{|c|}{ proposed } \\
\hline 31 & 2.71 & 2.26 & 2.51 & 1.00 \\
34 & 2.52 & 2.12 & 2.12 & 0.99 \\
37 & 2.33 & 1.99 & 1.91 & 1.00 \\
40 & 2.04 & 1.50 & 1.73 & 0.95 \\
\hline
\end{tabular}

TABLE V

$\Delta_{\text {PSNR }}[\mathrm{DB}]$ ON THE SIDE INFORMATION FOR GOP SIZE EQUAL TO 8 WITH RESPECT TO DISCOVER FOR HOMI AND FOR PROPOSED METHOD

equal to 4, and in Tab. VII for GOP size equal to 8 . As shown in [8], the improvement of SI in HOMI also improves the RD performance compared to DISCOVER. The method proposed here outperforms the performance of HOMI also in terms of rate distortion performance with respect to DISCOVER. The gain is slightly less than expected, because the bit allocation for each band is suboptimal. In fact, in the refinement step we use the same parity bits of the step 1 that are allocated for a different estimation. In spite of this, we can still achieve good profits in the decoded central WZFs as shown in Fig. 6 (with respect to Fig. 3). In fact for book arrival sequence we observe that the average PSNR of the lateral WZF is changed from $36.70 \mathrm{~dB}$ to $37.80 \mathrm{~dB}$. We remark that the total rate reduction is up to $13.04 \%$ w.r.t. the reference, with a PSNR improvement equal to $0.71 \mathrm{~dB}$ when GOP size is equal to 4 . While the total rate reduction is up to $12.27 \%$ with a PSNR improvement of $0.51 \mathrm{~dB}$ with respect to DISCOVER when the GOP size is equal to 8 . See also the RD curves in Fig. 7 and Fig. 8 .

\section{CONCLUSIONS AND FUTURE WORK}

We have observed that for a GOP size equal to 4 the central estimated WZFs have a lower average PSNR than the lateral ones. We propose to refine the SI on the central WZFs by using the lateral already decoded WZFs in order to interpolate frames closer than the KFs used initially for the estimation

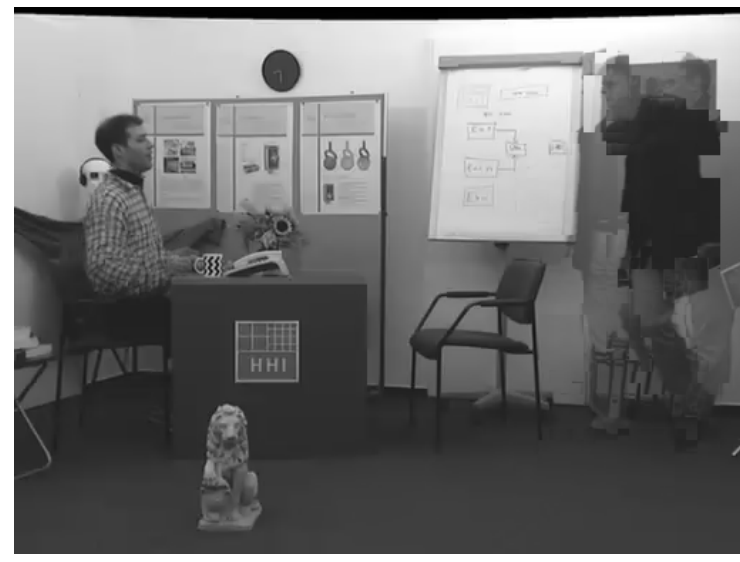

(a)

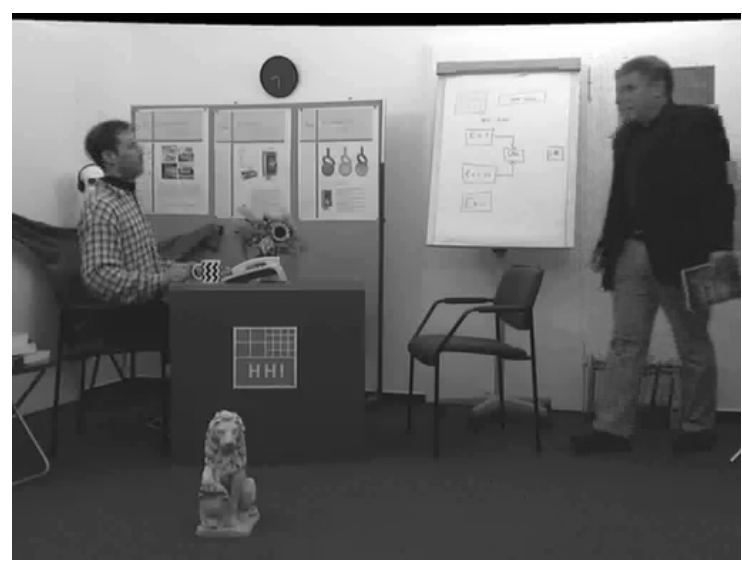

(b)

Fig. 5. Example of side information for GOP size equal to 8 with HOMI 5(a) and with the proposed method 5(b)

of the central WZF. The method used here for the motion interpolation is HOMI illustrated in [8]. We have also extended this method for GOP size equal to 8 . Our refinement method performs an improvement of the SI from $0.45 \mathrm{~dB}$ to $2.71 \mathrm{~dB}$ with respect to DISCOVER in the best case when the GOP size is equal to 8 . As future work we will propose to iterate this procedure for refining also the lateral WZFs until we reach a stable solution.

\section{REFERENCES}

[1] F. Dufaux, W. Gao, S. Tubaro, and A. Vetro, "Distributed video coding: Trends and perspectives," in EURASIP Journal on Image and Video Processing, 2009.

[2] D. Wyner and J. Ziv, "The rate-distortion function for source coding with side information at the decoder," in IEEE Transactions on Information Theory, vol. IT-22, 1976, pp. 1-10.

[3] A. Aaron, R. Zhang, and B. Girod, "Wyner-Ziv coding of motion video," in Asilomar Conference on Signals and Systems, Pacific Grove, California, Nov. 2002. 


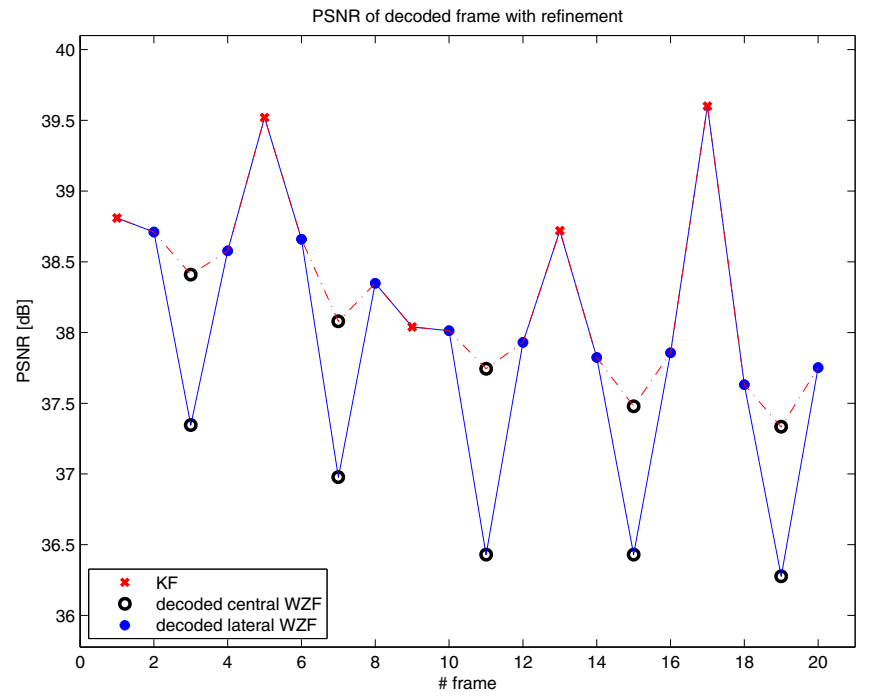

Fig. 6. PSNR of decoded frames before refinement (the blue line) and after refinement (the red dashed line) for book arrival $\mathrm{QP}=31$, GOP size $=4$

\begin{tabular}{|l|c|c|c|c|}
\hline & book arrival & ballet & jungle & breakdancer \\
\hline \hline \multicolumn{5}{|c|}{ HOMI } \\
\hline$\Delta_{\mathrm{R}}[\%]$ & -4.41 & -1.03 & -2.29 & 0.68 \\
$\Delta_{\mathrm{PSNR}}[\mathrm{dB}]$ & 0.20 & 0.05 & 0.11 & -0.02 \\
\hline \hline \multicolumn{5}{|c|}{ proposed } \\
\hline$\Delta_{\mathrm{R}}[\%]$ & -13.04 & -5.98 & -6.35 & 0.56 \\
$\Delta_{\mathrm{PSNR}}[\mathrm{dB}]$ & 0.56 & 0.30 & 0.29 & -0.00 \\
\hline
\end{tabular}

TABLE VI

RATE-DISTORTION ANALYSIS FOR HOMI AND FOR PROPOSED METHOD WITH RESPECT TO DISCOVER FOR GOP SIZE 4 BY CONSIDERING THE WHOLE VIDEO SEQUENCE

[4] A. Aaron, E. Setton, and B. Girod, "Towards practical Wyner-Ziv coding of video," in IEEE International Conf. on Image Processing, Barcelona, Spain, 2003.

[5] J. Ascenso, C. Brites, and F. Pereira, "Content adaptive Wyner-Ziv video coding driven by motion activity," in IEEE International Conf. on Image Processing, Atlanta, USA, 2006.

[6] C. Yaacoub, J. Farah, and B. Pesquet-Popescu, "New adaptive algorithms for GOP size control with return channel suppression in Wyner-Ziv video coding," International Journal of Digital Multimedia Broadcasting, 2009.

[7] X. Artigas, J. Ascenso, M. Dalai, S. Klomp, D. Kubasov, and M. Ouaret, "The Discover codec: Architecture, techniques and evaluation," Picture Coding Symposium, vol. 0, no. 0, nov 2007. [Online]. Available: /papers/data/2007/PCS-XAJAMDSKDKMO.pdf

[8] G. Petrazzuoli, M. Cagnazzo, and B. Pesquet-Popescu, "High order motion interpolation for side information improvement in DVC," in International Conference on Acoustics, Speech and Signal Processing, Dallas, TX, 2010.

[9] C. Brites, J. Ascenso, and F. Pereira, "Studying temporal correlation noise modeling for pixel based Wyner-Ziv video coding," in Image Processing, 2006 IEEE International Conference on, oct. 2006, pp. 273 $-276$.

[10] C. Brites and F. Pereira, "Correlation noise modeling for efficient pixel and transform domain Wyner-Ziv video coding," Circuits and Systems for Video Technology, IEEE Transactions on, vol. 18, no. 9, pp. 1177 -1190 , sept. 2008.

[11] G. Bjontegaard, "Calculation of average PSNR differences between RDcurves," in VCEG Meeting, Austin, USA, Apr. 2001.

\begin{tabular}{|l|c|c|c|c|}
\hline & book arrival & ballet & jungle & breakdancer \\
\hline \hline \multicolumn{5}{|c|}{ HOMI } \\
\hline$\Delta_{\mathrm{R}}[\%]$ & -6.64 & -1.74 & -4.31 & -3.50 \\
$\Delta_{\mathrm{PSNR}}[\mathrm{dB}]$ & 0.27 & 0.11 & 0.20 & 0.16 \\
\hline \hline \multicolumn{5}{|c|}{ proposed } \\
\hline$\Delta_{\mathrm{R}}[\%]$ & -12.27 & -3.19 & -10.34 & -4.57 \\
$\Delta_{\mathrm{PSNR}}[\mathrm{dB}]$ & 0.51 & 0.16 & 0.45 & 0.22 \\
\hline
\end{tabular}

TABLE VII

RATE-DISTORTION ANALYSIS FOR HOMI AND FOR PROPOSED METHOD WITH RESPECT TO DISCOVER FOR GOP SIZE 8 BY CONSIDERING THE WHOLE VIDEO SEQUENCE

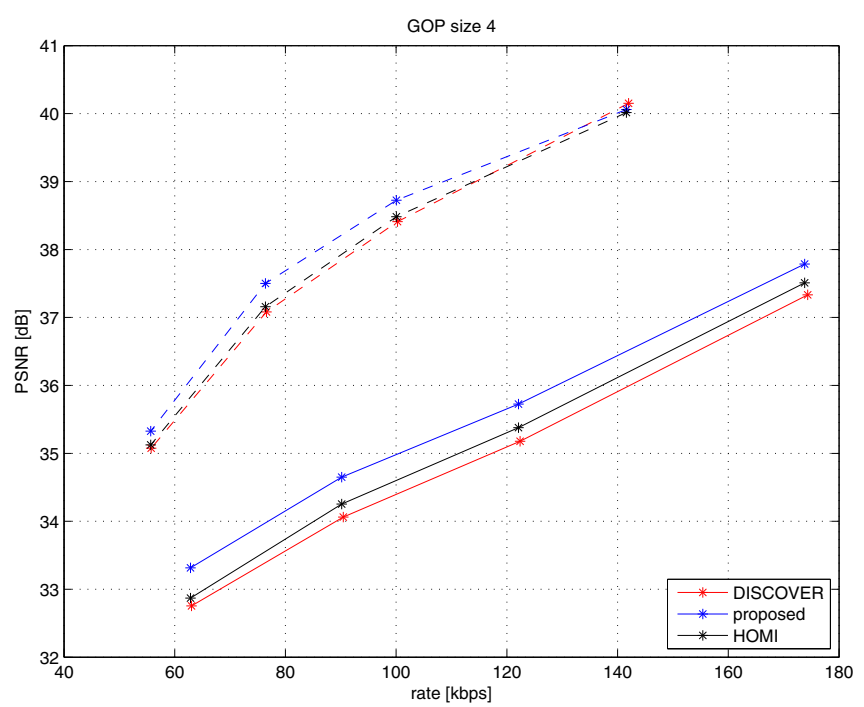

Fig. 7. Rate-distortion curve for book arrival (solid lines) and ballet (dashed lines) when GOP size is equal to 4

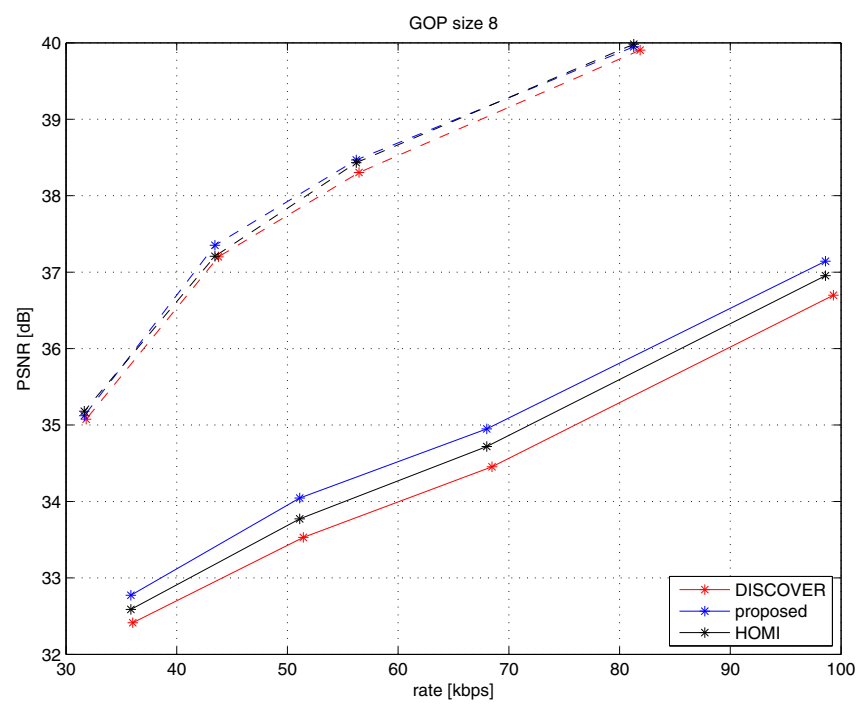

Fig. 8. Rate-distortion curve for book arrival (solid lines) and ballet (dashed lines) when GOP size is equal to 8 